\title{
Concerns and Preparedness for an Avian Influenza Pandemic: A Comparison between Community Hospital and Tertiary Hospital Healthcare Workers
}

\author{
Seng Kwing CHEONG ${ }^{1 *}$, Teck Yee WONG ${ }^{1}$, Heow Yong LEE$^{2}$, Yuke Tien FONG ${ }^{2}$, \\ Boon Yeow TAN ${ }^{3}$, Gerald $\mathrm{CH}_{\mathrm{KOH}}{ }^{1}$, Kin Ming $\mathrm{CHAN}^{4}$, \\ Sin Eng CHIA ${ }^{1}$ and David KOH ${ }^{1}$
}

\author{
${ }^{1}$ Department of Community, Occupational and Family Medicine, Yong Loo Lin School of Medicine, National \\ University of Singapore, Block MD3, 16 Medical Drive, 117597 Singapore \\ ${ }^{2}$ Department of Occupational Health and Epidemiology, Singapore General Hospital, Outram Road, 169608 Singapore \\ ${ }^{3}$ St Luke's Hospital, 2 Bukit Batok St 11, 659674 Singapore \\ ${ }^{4}$ Ang Mo Kio Thye Hua Kwan Hospital, 17 Ang Mo Kio Ave 9, 569766 Singapore
}

Received March 21, 2007 and accepted June 12, 2007

\begin{abstract}
Little is known about differences in levels of concerns or preparedness for an avian influenza (AI) pandemic among healthcare workers (HCWs) in different types of hospitals. We compared these concerns and preparedness between $326 \mathrm{HCWs}$ of two community hospitals (CHs) and 908 HCWs from a tertiary hospital (TH) using a self-administered questionnaire between March-June 2006. Response rates were $84.2 \%$ and $80.0 \%$ from the CHs and TH. Most HCWs (71.6\%) felt prepared for an AI outbreak and had significant concerns. They perceive an AI pandemic having adverse impacts on their personal life and work, such as people avoiding them (57.1\%). A greater percentage of TH compared to $\mathrm{CH}$ HCWs expressed concerns such as feeling their jobs put them at great AI exposure $(78.3 \%$ vs $67.5 \%, p=0.012)$. TH HCWs were more likely to report participating in readiness preparation activities, such as training for infection control $(90.0 \%$ vs $82.2 \%, p=0.014)$ and feel that they $(74.1 \%$ vs $64.7 \%, p=0.045)$ and their hospital $(86.8 \%$ vs $71.8 \%, p=0.000)$ were prepared for an outbreak. Healthcare institutions need to include personal, psychological and family concerns on the agenda and increase participation in readiness preparation activities among HCWs to help prepare for such future crises.
\end{abstract}

Key words: Avian influenza, Healthcare workers, Community hospital, Tertiary hospital, Preparedness, Concerns

\section{Introduction}

Despite significant medical gains of the last century, the danger posed by emerging infectious diseases has become even greater in our increasingly interconnected world ${ }^{1)}$. Singapore, an island republic with high standards of environmental hygiene, a comprehensive childhood immunization program, and strict control of imported food and livestock, has not been spared either. Since the 1990s,

*To whom correspondence should be addressed. a number of infectious diseases have emerged and re-emerged in Singapore, such as Nipah Virus infection in 19992), Severe Acute Respiratory Syndrome (SARS) in 2003, and dengue fever in the last few years. The increasing travel and trade within and beyond the region has been a significant contributing factor in this emergence ${ }^{3)}$.

It has been well documented that healthcare workers (HCWs) suffered from significant stress during the SARS outbreak $^{4)}$. As the frontline 'troops', they were, and will be at the forefront of danger in such infectious disease outbreaks. This was seen during the SARS epidemic, where a large 
proportion of outbreaks occurred in hospitals and one-fifth of probable SARS cases involved $\mathrm{HCWs}^{5}$. Another reason is that in an infectious outbreak, HCWs are more likely to be on physical and emotional overdrive because of an overstretched healthcare system ${ }^{6}$.

There is scant data on the differences in levels of concerns or preparedness among different types of hospitals. In Singapore, tertiary hospitals (THs) provide multi-disciplinary acute inpatient care, while community hospitals (CHs) provide intermediate healthcare for the convalescent sick and aged who do not require the care of the THs. With the looming threat of a global avian influenza (AI) pandemic, we decided to study and compare the concerns and preparedness for an $\mathrm{AI}$ pandemic among $\mathrm{HCW}$ s working in two CHs and a TH in Singapore.

\section{Subjects and Methods}

We administered an anonymous self-administered questionnaire to 1,524 $\mathrm{HCW}$ sorking at $2 \mathrm{CHs}$ and a $\mathrm{TH}$ in Singapore, from March through June 2006 (Fig. 1). The TH respondents comprised all the staff that participated in the respiratory protection program (respirator and Powered Air Purifying Respirators [PAPR] fitting sessions) and who held a wide range of jobs in the TH $(\mathrm{n}=1,137)$. At the two $\mathrm{CHs}$, we administered the questionnaire to all HCWs $(\mathrm{n}=387)$. Questionnaires were rejected if there were incomplete entries in at least two pages of the questionnaire, no demographic data, unreadable entries, or torn or missing pages.

The questionnaire was developed from an earlier survey used in a study of SARS and HCWs ${ }^{1}$. Participation was voluntary and ethics approval was provided by each hospital's Institutional Review Board.

The questionnaire included items about concerns regarding AI that were work-related ( 8 items) and nonwork-related (4 items), perceived impact of AI on personal life and work (10 items), and preparedness for AI outbreak at the workplace (16 items). These items were measured on a 6-point Likert scale for responses (strongly disagree, disagree, not sure but probably disagree, not sure but probably agree, agree, and strongly agree). During statistical analyses, we dichotomized this scale into positive response (strongly agree, agree, and probably agree) and negative response (strongly disagree, disagree, and probably disagree). We analyzed the data using SPSS Version 15.0 (SPSS Institute, Chicago, IL). Chi-square analysis was used to compare proportions and test for statistical significance.

\section{Results}

\section{Response rate and socio-demographic characteristics} (Table 1)

Overall, we obtained 1,234 valid responses. $326 \mathrm{HCWs}$ were from the $\mathrm{CHs}$ and $908 \mathrm{HCWs}$ were from the $\mathrm{TH}$. The response rate was $84.2 \%$ for the $\mathrm{CHs}$, and $80.0 \%$ for the TH. The mean age of HCWs was $31.3 \mathrm{yr}$ (standard deviation: $10.7 \mathrm{yr}$ ) and the median work experience was 2 $\mathrm{yr}$ (interquartile range: 1-7 yr). Females formed the majority in both CHs (83.4\%) and the TH surveyed (85.2\%). A total of $37.4 \%$ of the HCWs were married, and $59.2 \%$ were single.

\section{Work-related and non-work concerns (Table 2)}

Compared to $\mathrm{CH} \mathrm{HCWs}$, TH HCWs were likelier to feel that their jobs put them at great risk of exposure to $\mathrm{AI}(78.3 \%$ vs $67.5 \%, p=0.012$ ). More TH workers felt that they should not be looking after patients with AI $(26.3 \%$ vs $16.0 \%$, $p=0.001$ ) and reported that they might look for another job or consider resigning because of the risk of contracting AI (16.5\% vs $10.7 \%, p=0.027)$.

TH HCWs were also more concerned that people close to them were at high risk due to their occupation $(68.5 \%$ vs $56.1 \%, p=0.001)$; that people close to them would be worried for their health $(92.7 \%$ vs $81.9 \%, p=0.000)$ and that people close to them would be worried about getting infected by them $(85.4 \%$ vs $73.6 \%, p=0.001)$.

\section{Perceived impact on personal life and work (Table 2)}

In the event of a widespread AI outbreak, a higher proportion of $\mathrm{TH} \mathrm{HCWs}$ reported that they would be afraid of informing their family about their exposure risk (29.8\% vs $19.0 \%, p=0.001$ ) and avoid telling others about the nature of their work ( $27.0 \%$ vs $16.6 \%, p=0.000)$. A higher proportion of TH HCWs also reported that they feel that people would avoid them $(60.1 \%$ vs $48.5 \%, p=0.002)$ and their family members $(48.7 \%$ vs $40.2 \%, p=0.034)$ because of their occupation.

At work, TH HCWs were less likely to feel that there would be adequate staff to handle any increase in demand ( $53.6 \%$ vs $59.5 \%, p=0.002$ ) but more likely to feel that there could be more conflict amongst colleagues at work (38.2\% vs $30.1 \%, p=0.040$ ) and that they would have to work overtime $(72.8 \%$ vs $59.8 \%, p=0.001)$.

There were no differences in both groups regarding feeling more stressed at work, perceived increase in workload and doing work not normally done by themselves. 


\section{Concerns Regarding Bird Flu Outbreak}

Please rate (by Shading) the following statements to which you agree or disagree with it, on a scale of ' 1 ' to ' 6 ', ' 1 ' being 'Strongly Disagree' and ' 6 ' being 'Strongly Agree'.

\section{$\underline{\text { Work-Related Concerns }}$}

\begin{tabular}{|c|c|c|c|c|c|c|c|c|}
\hline & $\begin{array}{l}\text { In the event of a widespread bird } \\
\text { flu outbreak with an increased } \\
\text { risk of contracting bird flu myself: }\end{array}$ & $\begin{array}{l}\text { Strongly } \\
\text { Disagree }\end{array}$ & Disagree & $\begin{array}{l}\text { Not sure, } \\
\text { but } \\
\text { Probably } \\
\text { Disagree }\end{array}$ & $\begin{array}{l}\text { Not sure, } \\
\text { but } \\
\text { Probably } \\
\text { Agree }\end{array}$ & Agree & $\begin{array}{l}\text { Strongly } \\
\text { Agree }\end{array}$ & $\begin{array}{c}\text { Not } \\
\text { Applicable }\end{array}$ \\
\hline 1. & $\begin{array}{l}\text { I feel that my job would put me at } \\
\text { great risk of exposure to bird flu. }\end{array}$ & 0 & 0 & $\mathrm{O}$ & 0 & 0 & 0 & \\
\hline 2. & $\begin{array}{l}\text { I would be afraid of falling ill with } \\
\text { bird flu. }\end{array}$ & 0 & 0 & 0 & 0 & 0 & 0 & \\
\hline 3. & $\begin{array}{l}\text { I feel that I should not be looking } \\
\text { after patients with bird flu. }\end{array}$ & 0 & 0 & 0 & 0 & 0 & 0 & 0 \\
\hline 4. & $\begin{array}{l}\text { The risk I would be exposed to at } \\
\text { work is not acceptable. }\end{array}$ & 0 & 0 & $\mathrm{O}$ & 0 & 0 & $\mathrm{O}$ & \\
\hline 5. & $\begin{array}{l}\text { I would accept that the risk of } \\
\text { contracting bird flu is part of my job. }\end{array}$ & 0 & 0 & 0 & 0 & 0 & 0 & \\
\hline 6. & $\begin{array}{l}\text { I might look for another job or } \\
\text { consider resigning because of the } \\
\text { risk of contracting bird flu. }\end{array}$ & 0 & O & 0 & 0 & $\mathrm{O}$ & 0 & \\
\hline 7. & $\begin{array}{l}\text { I would consider it acceptable if my } \\
\text { colleagues resign because of their } \\
\text { fear of bird flu. }\end{array}$ & $\mathrm{O}$ & O & $\mathrm{O}$ & 0 & $\mathrm{O}$ & 0 & \\
\hline 8. & $\begin{array}{l}\text { I am confident that my employer } \\
\text { would look after my medical needs } \\
\text { if I were to fall ill with bird flu. }\end{array}$ & $\mathrm{O}$ & O & $\mathrm{O}$ & $\mathrm{O}$ & $\mathrm{O}$ & $\mathrm{O}$ & \\
\hline
\end{tabular}

\section{Non-Work Concerns}

\begin{tabular}{|c|c|c|c|c|c|c|c|c|}
\hline & $\begin{array}{l}\text { With regard to the possibility of } \\
\text { passing bird flu to those closest to } \\
\text { me (spouse/partner, family } \\
\text { members, friends etc): }\end{array}$ & $\begin{array}{l}\text { Strongly } \\
\text { Disagree }\end{array}$ & Disagree & $\begin{array}{l}\text { Not sure, } \\
\text { but } \\
\text { Probably } \\
\text { Disagree }\end{array}$ & $\begin{array}{l}\text { Not sure, } \\
\text { but } \\
\text { Probably } \\
\text { Agree }\end{array}$ & Agree & $\begin{array}{l}\text { Strongly } \\
\text { Agree }\end{array}$ & $\begin{array}{c}\text { Not } \\
\text { Applicable }\end{array}$ \\
\hline 9. & $\begin{array}{l}\text { People close to me would be at high } \\
\text { risk of getting bird flu because of } \\
\text { my job. }\end{array}$ & $\mathrm{O}$ & $\mathrm{O}$ & $\mathrm{O}$ & $\mathrm{O}$ & $\mathrm{O}$ & $\mathrm{O}$ & \\
\hline 10. & I would be most concerned for my: & & & & & & & \\
\hline a. & Spouse/partner. & $\mathrm{O}$ & $\mathrm{O}$ & $\mathrm{O}$ & $\mathrm{O}$ & $\mathrm{O}$ & $\mathrm{O}$ & $\mathrm{O}$ \\
\hline b. & Parents. & $\mathrm{O}$ & $\mathrm{O}$ & $\mathrm{O}$ & $\mathrm{O}$ & $\mathrm{O}$ & $\mathrm{O}$ & $\mathrm{O}$ \\
\hline c. & Children. & $\mathrm{O}$ & $\mathrm{O}$ & $\mathrm{O}$ & $\mathrm{O}$ & $\mathrm{O}$ & $\mathrm{O}$ & $\mathrm{O}$ \\
\hline d. & Close friends. & $\mathrm{O}$ & $\mathrm{O}$ & $\mathrm{O}$ & $\mathrm{O}$ & $\mathrm{O}$ & $\mathrm{O}$ & $\mathrm{O}$ \\
\hline e. & Work Colleagues. & $\mathrm{O}$ & $\mathrm{O}$ & $\mathrm{O}$ & $\mathrm{O}$ & $\mathrm{O}$ & 0 & 0 \\
\hline f. & Others & 0 & 0 & 0 & 0 & 0 & 0 & 0 \\
\hline 11. & $\begin{array}{l}\text { People close to me would be worried } \\
\text { for my health. }\end{array}$ & 0 & 0 & 0 & 0 & 0 & 0 & \\
\hline 12. & $\begin{array}{l}\text { People close to me would be worried } \\
\text { as they may get infected by me. }\end{array}$ & 0 & 0 & 0 & 0 & $\mathrm{O}$ & $\mathrm{O}$ & \\
\hline
\end{tabular}

Fig. 1. Sections of the Questionnaire on Attitudes, Concerns, and Preparedness of Healthcare Workers with regard to a Bird Flu Outbreak. 


\section{Preparedness for a bird flu pandemic (Table 3)}

The overall prevalence of perceived preparedness in both groups was high. $71.6 \%$ of the HCWs who responded felt that they were personally prepared while $82.8 \%$ felt that their institutions were prepared. Nevertheless, TH HCWs were more likely to feel personally prepared $(74.1 \%$ vs $64.7 \%, p=0.045$ ) and more likely to report that their hospital was prepared $(86.8 \%$ vs $71.8 \%, p=0.000)$.

The majority of HCWs had received training for infection control $(87.9 \%)$; knew the hospital had an outbreak preparedness plan $(87.2 \%)$ and have been informed of that plan (77.8\%). A greater percentage of $\mathrm{TH} \mathrm{HCW}$ s reported having had training $(90.0 \%$ vs $82.2 \%, p=0.014)$, knew that their institution has a preparedness plan $(91.4 \%$ vs $75.5 \%$, $p=0.000)$ and were informed of the plan $(82.3 \%$ vs $65.3 \%$, $p=0.000)$.

In the six months prior to our survey, TH HCWs were likelier to have attended infection control related meetings (59.8\% vs $50.6 \%, p=0.039)$, received influenza vaccination $(82.5 \%$ vs $63.8 \%, p=0.000)$ and received adequate training in use of personal protective equipment $(81.5 \%$ vs $71.8 \%$, $p=0.032$ ).

\section{Discussion}

\section{Demographic factors}

Females formed the majority of respondents in both $\mathrm{CHs}$ and TH surveyed due to the large proportion of nurses, who are predominantly female in Singapore.

\section{Concerns}

As a combined group, $\mathrm{CH}$ and $\mathrm{TH} \mathrm{HCW}$ perceived a great risk of personal exposure to the bird flu virus from their job and feared falling ill with the disease. This is consistent with a study which found that danger to personal health was one of the most prevalent fears among HCWs who experienced significant psychological stress during the SARS crisis ${ }^{4}$.

HCWs are likely to be as, if not more, concerned than the average citizen because they probably have a greater understanding of the associated risks. Fatalities related to SARS among HCWs who were exposed and infected occupationally also contribute to employees' fears regarding potentially fatal infectious diseases ${ }^{7)}$. $38.7 \%$ of HCWs surveyed had at least $4 \mathrm{yr}$ of work experience in healthcare which suggests that many participants had past experience working during the SARS outbreak in 2003. Moreover, SARS affected all Singaporeans and most HCWs would likely remember and be affected by it as there was much
Table 1. Demographic characteristics of study participants

\begin{tabular}{|c|c|c|c|}
\hline Characteristics & $\begin{array}{c}\text { TH N=908 } \\
\text { n }(\%)\end{array}$ & $\begin{array}{c}\mathrm{CH} \mathrm{N}=326 \\
\mathrm{n}(\%)\end{array}$ & $\begin{array}{c}\text { Total } \mathrm{N}=1,234 \\
\mathrm{n}(\%)\end{array}$ \\
\hline \multicolumn{4}{|l|}{ Ethnicity* } \\
\hline Chinese & $494(54.4)$ & $142(43.6)$ & $636(51.5)$ \\
\hline Malay & $175(19.3)$ & $21(6.4)$ & $196(15.9)$ \\
\hline Indian & $122(13.4)$ & $23(7.1)$ & $145(11.8)$ \\
\hline Others & $110(12.1)$ & 104 (31.9) & $214(17.3)$ \\
\hline \multicolumn{4}{|l|}{ Age $(y r)^{*}$} \\
\hline$<20$ & $21(2.3)$ & $0(0.0)$ & $21(1.7)$ \\
\hline $20-29$ & $579(63.8)$ & $66(20.2)$ & $645(52.3)$ \\
\hline $30-39$ & $150(16.5)$ & $134(41.1)$ & $284(23.0)$ \\
\hline $40-49$ & $66(7.3)$ & $53(16.3)$ & $119(9.6)$ \\
\hline 50 and older & $57(6.3)$ & $46(14.1)$ & $103(8.3)$ \\
\hline \multicolumn{4}{|l|}{ Sex* } \\
\hline Male & $131(14.4)$ & $42(12.9)$ & $173(14.0)$ \\
\hline Female & 774 (85.2) & $272(83.4)$ & $1,046(84.8)$ \\
\hline \multicolumn{4}{|l|}{ Job Title* } \\
\hline Doctor & $51(5.6)$ & $7(2.1)$ & $58(4.7)$ \\
\hline Nurse & $569(62.7)$ & $197(60.4)$ & $766(62.1)$ \\
\hline $\begin{array}{l}\text { Others (e.g. clerks, } \\
\text { allied health) }\end{array}$ & $288(31.7)$ & $104(32.0)$ & $392(31.8)$ \\
\hline \multicolumn{4}{|l|}{ Marital Status* } \\
\hline Married & 297 (32.7) & $164(50.3)$ & $461(37.4)$ \\
\hline Single & $583(64.2)$ & $148(45.4)$ & $731(59.2)$ \\
\hline $\begin{array}{l}\text { Divorced/Separated } \\
\text { /Widowed }\end{array}$ & $25(2.8)$ & $2(0.6)$ & $27(2.2)$ \\
\hline \multicolumn{4}{|l|}{$\begin{array}{l}\text { Experience as a healthcare } \\
\text { worker (Years) }\end{array}$} \\
\hline$<4$ & $640(70.5)$ & $116(35.6)$ & $756(61.3)$ \\
\hline$\geq 4$ & $268(29.5)$ & $210(64.4)$ & $478(38.7)$ \\
\hline
\end{tabular}

* Numbers for each item may not add up to the total number of HCWs in the $\mathrm{CH}$ or $\mathrm{TH}$ because of missing values.

publicity during that period.

A greater proportion of TH HCWs as compared to $\mathrm{CH}$ HCWs were concerned of the risk to themselves and those close to them because of their job as a $\mathrm{HCW}$ in an AI pandemic. One reason why $\mathrm{CH} \mathrm{HCW}$ may be less likely to be concerned is because patients in $\mathrm{CHs}$ are likely to have less acute medical conditions. CHs serve as intermediate care facilities which cater to patients who are fit for discharge from acute hospitals (including THs), but require inpatient convalescent and rehabilitative care ${ }^{8)}$. They provide the transitional interface between acute hospitals and the community for patients who no longer require the intensive and specialized care provided by acute hospitals, yet are not ready to be returned to the community for medical or social reasons. These are commonly poststroke or post-fracture patients who require rehabilitation and elderly patients who need longer convalescence after 
Table 2. HCW concerns and perceived impact on personal life and work regarding a bird flu pandemic

\begin{tabular}{|c|c|c|c|c|}
\hline \multirow{2}{*}{$\begin{array}{l}\text { Concerns Regarding } \\
\text { a Bird Flu Pandemic }\end{array}$} & $\mathrm{TH} \mathrm{n}=908$ & $\mathrm{CH} \mathrm{n}=326$ & Total $n=1,234$ & \multirow[b]{2}{*}{$p$ value } \\
\hline & $\underset{\%}{\text { Agree }}$ & $\underset{\%}{\text { Agree }}$ & $\underset{\%}{\text { Agree }}$ & \\
\hline \multicolumn{5}{|l|}{ Work-Related Concerns } \\
\hline My job would put me at great exposure risk & $\begin{array}{c}711 \\
(78.3 \%)\end{array}$ & $\begin{array}{c}220 \\
(67.5 \%)\end{array}$ & $\begin{array}{c}931 \\
(75.4 \%)\end{array}$ & 0.012 \\
\hline I am afraid of falling ill with bird flu & $\begin{array}{c}659 \\
(72.6 \%)\end{array}$ & $\begin{array}{c}232 \\
(71.2 \%)\end{array}$ & $\begin{array}{c}891 \\
(72.2 \%)\end{array}$ & 0.359 \\
\hline I should not be looking after bird flu patients & $\begin{array}{c}239 \\
(26.3 \%)\end{array}$ & $\begin{array}{c}52 \\
(16.0 \%)\end{array}$ & $\begin{array}{c}291 \\
(23.6 \%)\end{array}$ & 0.001 \\
\hline Risk is not acceptable & $\begin{array}{c}245 \\
(27.0 \%)\end{array}$ & $\begin{array}{c}67 \\
(20.6 \%)\end{array}$ & $\begin{array}{c}312 \\
(25.3 \%)\end{array}$ & 0.074 \\
\hline Accept that risk is part of job & $\begin{array}{c}656 \\
(72.2 \%)\end{array}$ & $\begin{array}{c}217 \\
(66.6 \%)\end{array}$ & $\begin{array}{c}873 \\
(70.7 \%)\end{array}$ & 0.410 \\
\hline Might look for another job because of risk & $\begin{array}{c}150 \\
(16.5 \%)\end{array}$ & $\begin{array}{c}35 \\
(10.7 \%)\end{array}$ & $\begin{array}{c}185 \\
(15.0 \%)\end{array}$ & 0.027 \\
\hline Acceptable if colleagues resign because of their fear & $\begin{array}{c}437 \\
(48.1 \%)\end{array}$ & $\begin{array}{c}152 \\
(46.6 \%)\end{array}$ & $\begin{array}{c}589 \\
(47.7 \%)\end{array}$ & 0.925 \\
\hline $\begin{array}{l}\text { Confident employer would look after my } \\
\text { needs if I fall ill with bird flu }\end{array}$ & $\begin{array}{c}803 \\
(88.4 \%)\end{array}$ & $\begin{array}{c}289 \\
(88.7 \%)\end{array}$ & $\begin{array}{c}1,092 \\
(88.5 \%)\end{array}$ & 0.036 \\
\hline \multicolumn{5}{|l|}{ Non-Work Concerns } \\
\hline $\begin{array}{l}\text { People close to me would be at high risk of } \\
\text { getting bird flu because of my job }\end{array}$ & $\begin{array}{c}622 \\
(68.5 \%)\end{array}$ & $\begin{array}{c}183 \\
(56.1 \%)\end{array}$ & $\begin{array}{c}805 \\
(65.2 \%)\end{array}$ & 0.001 \\
\hline $\begin{array}{l}\text { I would be most concerned for my: } \\
\text { Spouse/partner }\end{array}$ & $\begin{array}{c}627 \\
(69.1 \%)\end{array}$ & $\begin{array}{c}213 \\
(65.3 \%)\end{array}$ & $\begin{array}{c}840 \\
(68.1 \%)\end{array}$ & 0.303 \\
\hline Parents & $\begin{array}{c}766 \\
(84.4 \%)\end{array}$ & $\begin{array}{c}239 \\
(73.3 \%)\end{array}$ & $\begin{array}{c}1,005 \\
(81.4 \%)\end{array}$ & 0.024 \\
\hline Children & $\begin{array}{c}452 \\
(49.8 \%)\end{array}$ & $\begin{array}{c}178 \\
(54.6 \%)\end{array}$ & $\begin{array}{c}630 \\
(51.1 \%)\end{array}$ & 0.211 \\
\hline Close friends & $\begin{array}{c}809 \\
(89.1 \%)\end{array}$ & $\begin{array}{c}254 \\
(77.9 \%)\end{array}$ & $\begin{array}{c}1,063 \\
(86.1 \%)\end{array}$ & 0.000 \\
\hline Work Colleagues & $\begin{array}{c}846 \\
(93.2 \%)\end{array}$ & $\begin{array}{c}273 \\
(83.7 \%)\end{array}$ & $\begin{array}{c}1,119 \\
(90.7 \%)\end{array}$ & 0.000 \\
\hline Others & $\begin{array}{c}306 \\
(33.7 \%)\end{array}$ & $\begin{array}{c}102 \\
(31.3 \%)\end{array}$ & $\begin{array}{l}1,119 \\
(90.7 \%)\end{array}$ & 0.221 \\
\hline People close to me would be worried for my health & $\begin{array}{c}842 \\
(92.7 \%)\end{array}$ & $\begin{array}{c}267 \\
(81.9 \%)\end{array}$ & $\begin{array}{c}1,109 \\
(89.9 \%)\end{array}$ & 0.000 \\
\hline $\begin{array}{l}\text { People close to me would be worried as } \\
\text { they may get infected by me }\end{array}$ & $\begin{array}{c}775 \\
(85.4 \%)\end{array}$ & $\begin{array}{c}240 \\
(73.6 \%)\end{array}$ & $\begin{array}{c}1,015 \\
(82.3 \%)\end{array}$ & 0.001 \\
\hline \multirow{2}{*}{$\begin{array}{l}\text { Perceived Impact Regarding a Bird Flu } \\
\text { Pandemic on Personal Life and Work }\end{array}$} & TH n=908 & $\mathrm{CH} \mathrm{n}=326$ & Total $n=1,234$ & \multirow[b]{2}{*}{$p$ value } \\
\hline & $\underset{\%}{\text { Agree }}$ & $\underset{\%}{\text { Agree }}$ & $\underset{\%}{\text { Agree }}$ & \\
\hline $\begin{array}{l}\text { I would be afraid of telling my family } \\
\text { about the risk I am exposed to }\end{array}$ & $\begin{array}{c}271 \\
(29.8 \%)\end{array}$ & $\begin{array}{c}62 \\
(19.0 \%)\end{array}$ & $\begin{array}{c}333 \\
(27.0 \%)\end{array}$ & 0.001 \\
\hline People would avoid me because of my job & $\begin{array}{c}546 \\
(60.1 \%)\end{array}$ & $\begin{array}{c}158 \\
(48.5 \%)\end{array}$ & $\begin{array}{c}704 \\
(57.1 \%)\end{array}$ & 0.002 \\
\hline $\begin{array}{l}\text { People would avoid my family members } \\
\text { because of my job }\end{array}$ & $\begin{array}{c}442 \\
(48.7 \%)\end{array}$ & $\begin{array}{c}131 \\
(40.2 \%)\end{array}$ & $\begin{array}{c}573 \\
(46.4 \%)\end{array}$ & 0.034 \\
\hline $\begin{array}{l}\text { I would avoid telling other people } \\
\text { about the nature of my job }\end{array}$ & $\begin{array}{c}245 \\
(27.0 \%)\end{array}$ & $\begin{array}{c}54 \\
(16.6 \%)\end{array}$ & $\begin{array}{c}299 \\
(24.2 \%)\end{array}$ & 0.000 \\
\hline $\begin{array}{l}\text { There would be adequate staff at my workplace } \\
\text { to handle the increased demand }\end{array}$ & $\begin{array}{c}487 \\
(53.6 \%)\end{array}$ & $\begin{array}{c}194 \\
(59.5 \%)\end{array}$ & $\begin{array}{c}681 \\
(55.2 \%)\end{array}$ & 0.002 \\
\hline $\begin{array}{l}\text { There would be more conflict } \\
\text { amongst colleagues at work }\end{array}$ & $\begin{array}{c}347 \\
(38.2 \%)\end{array}$ & $\begin{array}{c}98 \\
(30.1 \%)\end{array}$ & $\begin{array}{c}445 \\
(36.1 \%)\end{array}$ & 0.040 \\
\hline I would feel more stressed at work & $\begin{array}{c}594 \\
(65.4 \%)\end{array}$ & $\begin{array}{c}199 \\
(61.0 \%)\end{array}$ & $\begin{array}{c}793 \\
(64.3 \%)\end{array}$ & 0.517 \\
\hline I would have an increase in workload & $\begin{array}{c}676 \\
(74.4 \%)\end{array}$ & $\begin{array}{c}232 \\
(71.2 \%)\end{array}$ & $\begin{array}{c}908 \\
(73.6 \%)\end{array}$ & 0.782 \\
\hline I would have to work overtime & $\begin{array}{c}661 \\
(72.8 \%)\end{array}$ & $\begin{array}{c}195 \\
(59.8 \%)\end{array}$ & $\begin{array}{c}856 \\
(69.4 \%)\end{array}$ & 0.001 \\
\hline $\begin{array}{l}\text { I would have to do work } \\
\text { not normally done by me }\end{array}$ & $\begin{array}{c}664 \\
(73.1 \%)\end{array}$ & $\begin{array}{c}211 \\
(64.7 \%)\end{array}$ & $\begin{array}{c}875 \\
(70.9 \%)\end{array}$ & 0.101 \\
\hline
\end{tabular}

* Numbers for each item may not add up to the total number of HCWs in the $\mathrm{CH}$ or $\mathrm{TH}$ because of missing values. 
Table 3. Preparedness for a bird flu pandemic

\begin{tabular}{|c|c|c|c|c|}
\hline & $\begin{array}{c}\text { TH } \mathrm{n}=908 \\
\text { Agree } \\
\%\end{array}$ & $\begin{array}{c}\mathrm{CH} \mathrm{n}=326 \\
\text { Agree } \\
\%\end{array}$ & $\begin{array}{c}\text { Total } \mathrm{n}=1,234 \\
\text { Agree } \\
\%\end{array}$ & $p$ value \\
\hline $\begin{array}{l}\text { There is an infection control } \\
\text { committee in my clinic }\end{array}$ & $\begin{array}{c}850 \\
(93.6 \%)\end{array}$ & $\begin{array}{c}293 \\
(89.9 \%)\end{array}$ & $\begin{array}{c}1,143 \\
(92.6 \%)\end{array}$ & 0.569 \\
\hline $\begin{array}{l}\text { I have received training for } \\
\text { infection control by my clinic }\end{array}$ & $\begin{array}{c}817 \\
(90.0 \%)\end{array}$ & $\begin{array}{c}268 \\
(82.2 \%)\end{array}$ & $\begin{array}{c}1,085 \\
(87.9 \%)\end{array}$ & 0.014 \\
\hline $\begin{array}{l}\text { I have been recommended by my } \\
\text { clinic to receive flu vaccination }\end{array}$ & $\begin{array}{c}858 \\
(94.5 \%)\end{array}$ & $\begin{array}{c}266 \\
(81.6 \%)\end{array}$ & $\begin{array}{c}1,124 \\
(91.1 \%)\end{array}$ & 0.000 \\
\hline $\begin{array}{l}\text { There is infection control staff } \\
\text { in my clinic }\end{array}$ & $\begin{array}{c}871 \\
(95.9 \%)\end{array}$ & $\begin{array}{c}300 \\
(92.0 \%)\end{array}$ & $\begin{array}{c}1,171 \\
(94.9 \%)\end{array}$ & 0.789 \\
\hline $\begin{array}{l}\text { My clinic has a preparedness plan } \\
\text { for a bird flu outbreak }\end{array}$ & $\begin{array}{c}830 \\
(91.4 \%)\end{array}$ & $\begin{array}{c}246 \\
(75.5 \%)\end{array}$ & $\begin{array}{c}1,076 \\
(87.2 \%)\end{array}$ & 0.000 \\
\hline $\begin{array}{l}\text { My clinic has informed me of their } \\
\text { bird flu outbreak preparedness plan }\end{array}$ & $\begin{array}{c}747 \\
(82.3 \%)\end{array}$ & $\begin{array}{c}213 \\
(65.3 \%)\end{array}$ & $\begin{array}{c}960 \\
(77.8 \%)\end{array}$ & 0.000 \\
\hline $\begin{array}{l}\text { My clinic is prepared for a bird flu } \\
\text { outbreak }\end{array}$ & $\begin{array}{c}788 \\
(86.8 \%)\end{array}$ & $\begin{array}{c}234 \\
(71.8 \%)\end{array}$ & $\begin{array}{c}1,022 \\
(82.8 \%)\end{array}$ & 0.000 \\
\hline $\begin{array}{l}\text { I am personally prepared for } \\
\text { a bird flu outbreak }\end{array}$ & $\begin{array}{c}673 \\
(74.1 \%)\end{array}$ & $\begin{array}{c}211 \\
(64.7 \%)\end{array}$ & $\begin{array}{c}884 \\
(71.6 \%)\end{array}$ & 0.045 \\
\hline $\begin{array}{l}\text { I have attended infection control } \\
\text { training sessions }\end{array}$ & $\begin{array}{c}703 \\
(77.4 \%)\end{array}$ & $\begin{array}{c}239 \\
(73.3 \%)\end{array}$ & $\begin{array}{c}942 \\
(76.3 \%)\end{array}$ & 0.759 \\
\hline $\begin{array}{l}\text { I have participated in infection } \\
\text { control audits }\end{array}$ & $\begin{array}{c}574 \\
(63.2 \%)\end{array}$ & $\begin{array}{c}181 \\
(55.5 \%)\end{array}$ & $\begin{array}{c}755 \\
(61.2 \%)\end{array}$ & 0.122 \\
\hline $\begin{array}{l}\text { I have attended infection control } \\
\text { related meetings }\end{array}$ & $\begin{array}{c}543 \\
(59.8 \%)\end{array}$ & $\begin{array}{c}165 \\
(50.6 \%)\end{array}$ & $\begin{array}{c}708 \\
(57.4 \%)\end{array}$ & 0.039 \\
\hline I have received flu vaccination & $\begin{array}{c}749 \\
(82.5 \%)\end{array}$ & $\begin{array}{c}208 \\
(63.8 \%)\end{array}$ & $\begin{array}{c}957 \\
(77.6 \%)\end{array}$ & 0.000 \\
\hline Bought anti-bird flu medication & $\begin{array}{c}58 \\
(6.4 \%)\end{array}$ & $\begin{array}{c}8 \\
(2.5 \%)\end{array}$ & $\begin{array}{c}66 \\
(5.3 \%)\end{array}$ & 0.011 \\
\hline Bought masks & $\begin{array}{c}105 \\
(11.6 \%)\end{array}$ & $\begin{array}{c}41 \\
(12.6 \%)\end{array}$ & $\begin{array}{c}146 \\
(11.8 \%)\end{array}$ & 0.393 \\
\hline $\begin{array}{l}\text { Received adequate personal } \\
\text { protective equipment training }\end{array}$ & $\begin{array}{c}740 \\
(81.5 \%)\end{array}$ & $\begin{array}{c}234 \\
(71.8 \%)\end{array}$ & $\begin{array}{c}974 \\
(78.9 \%)\end{array}$ & 0.032 \\
\hline $\begin{array}{l}\text { Have someone to turn to if unsure of } \\
\text { use of personal protective equipment }\end{array}$ & $\begin{array}{c}810 \\
(89.2 \%)\end{array}$ & $\begin{array}{c}262 \\
(80.4 \%)\end{array}$ & $\begin{array}{c}1,072 \\
(86.9 \%)\end{array}$ & 0.106 \\
\hline
\end{tabular}

*Numbers for each item may not add up to the total number of HCWs in the $\mathrm{CH}$ or $\mathrm{TH}$ because of missing values.

acute illness ${ }^{9)}$. Thus, patients who are admitted to CHs are 'screened', coming from THs, whereas those admitted to THs come 'unscreened' from the community. Another reason may be that as criteria for admission to $\mathrm{CHs}$, patients who are medically unstable or have medical problems which are inadequately worked up are not discharged to the $\mathrm{CHs}$ but remain at the THs. This essentially screens out potential patients ill with $\mathrm{AI}$ in an outbreak. Thus $\mathrm{CH}$ HCWs perceive themselves to be at less exposure risk compared to $\mathrm{TH}$ HCWs.

Despite HCWs' concerns, it is heartening to note that the majority ( $83.5 \%$ of TH HCWs and $89.3 \%$ of $\mathrm{CH} \mathrm{HCWs})$ would not consider leaving their job but would continue to look after patients during an AI outbreak. This is similar to a study of 60 general practitioners in Australia, in which all of them expressed willingness to provide professional services in an influenza pandemic, citing as reasons strong personal work ethic, a sense of personal responsibility for their patients' welfare, and viewing not working as an abandonment of their responsibilities to their patients and colleagues ${ }^{10)}$.

However, not all studies have found that HCWs were willing to continue working in an infectious disease outbreak. Qureshi et al. reported that HCWs were less willing to report for duty during infectious diseases epidemics (e.g. SARS, smallpox) than for most other forms of catastrophic disasters (e.g. environmental disasters, mass casualty incidents) ${ }^{11)}$. In another study on public health workers, Balicer et al. 
found that almost half of HCWs were unlikely to report to duty during an influenza pandemic ${ }^{12)}$. In a study by Ruderman et al. after the SARS outbreak, healthcare professionals questioned their choice of career and decided to leave their profession and pursue new ventures, showing an unwillingness or inability to care for patients in the face of risk $^{13)}$. Reasons for such behavior probably reflect the complexity of the issue of caring for others under conditions of great personal risk. HCWs express conflict between their roles as health care provider and parent, feeling on one hand altruism and professional responsibility and, on the other hand, fear and guilt about potentially exposing their families to infection ${ }^{14)}$.

Although the majority felt that they should stay, almost half of our respondents ( $48.1 \%$ of TH HCWs and $46.6 \%$ of $\mathrm{CH} \mathrm{HCWs}$ ) felt it acceptable if their colleagues resigned out of fear. A possible reason is that these respondents placed the anxiety of their colleagues above their own. Although perceived employer support was a protective factor against stress in other studies ${ }^{4}$, we did not find a significant association in our study. A possible reason is that most HCWs $(88.5 \%)$ were already confident that their employer will look after their needs if they fell ill with AI.

\section{Perceived impact on personal life and work}

Another area of concern we found was characteristic of HCWs dealing with infectious diseases: many respondents felt that they would experience social stigmatization and ostracism, which was also reported in other studies ${ }^{6}$. This was more obvious in TH HCWs who were more likely to fear telling their family about their risk of exposure, feel that people would avoid them or their family due to their job, and avoid telling others about their job nature.

Studies have also found that concern for personal or family health among HCWs was significantly associated with personal or family lifestyles being affected by SARS and being treated differently by people because of working in a SARS-affected hospital ${ }^{15}$. Our results are also consistent with other studies that found that most HCWs have greater stress and workload, work overtime and do work normally done by others during an infectious disease outbreak ${ }^{1,4)}$.

A higher percentage of TH HCWs felt that there would be more conflict amongst colleagues at work and the need to work overtime. On the other hand, a higher percentage of $\mathrm{CH}$ HCWs felt that there would be adequate staff at the workplace to handle the increased demand. Again, the greater intensity of work and severity of disease of patients in THs probably account for this difference.

\section{Preparedness for a bird flu pandemic}

Singapore, together with many other countries, has been developing preparedness plans ${ }^{16)}$ due to increasing concern of an imminent influenza pandemic worldwide. As a result of the lessons learnt from SARS, Singapore has further strengthened its operational readiness and laboratory safety to respond to SARS, AI and other emerging diseases ${ }^{3)}$. Control measures used for SARS have been adapted for use to mitigate the spread of influenza, including infection control measures instituted at healthcare institutions, border health and community-wide measures and quarantine measure ${ }^{17)}$.

Many health measures are already in place, such as enhanced disease surveillance capability, increased isolation facilities in hospitals and, stockpiles of anti-viral drugs and increasing isolation facilities in hospitals. If required, the Singapore authorities will complement these with border control measures in order to help reduce the spread of influenza to Singapore. Singapore also works closely with other countries and with the relevant world health authorities to monitor and share outbreak information.

These comprehensive preparatory measures are probably the reasons why a high proportion of our HCWs reported themselves and their hospitals being prepared for an AI pandemic.

At the hospital level, infection control activities are in place, including training sessions and related meetings, having a hospital AI outbreak preparedness plan, and informing HCWs of such plans, and training in use of personal protective equipment (PPE). These activities were found to be positively associated with $\mathrm{HCWs}$ ' personal preparedness for an AI outbreak. This is expected as any activity that imparts knowledge and skills about a disease and its prevention naturally prepares one to better deal with it. TH HCWs compared to CH HCWs were likelier to report having participated in such activities. This may be a reason why they were also likelier to report that they and their hospital were prepared for an AI outbreak.

In contrast, a Canadian study of Ottawa family physicians' preparedness for public health emergencies found that only about a quarter of respondents felt prepared for a respiratory epidemic, with almost half feeling unprepared for an emergency ${ }^{18}$. A possible reason is that respondents in Ottawa have had no first-hand experience of a respiratory infectious disease outbreak as the SARS epidemic in Canada mainly affected the city of Toronto. Singapore, on the other hand, has had first-hand experience of SARS in 2003, with our country having 238 cases and 33 deaths, out of which 5 were $\mathrm{HCWs}^{11}$.

Greater psychosocial support of HCWs may be required, 
as suggested by other studies which have concluded that more appropriate psychosocial support is needed to prevent HCWs burning out from overwork and high stress during an infectious disease outbreak ${ }^{6,15}$. As pointed out by Qureshi et al., the most effective methods to allay fears and concerns for personal safety are probably HCW education, provision of appropriate PPE, and assurance of effective environmental controls ${ }^{11)}$. There is a need to put psychological concerns and stresses of HCWs on the agenda when preparing for an AI pandemic because there is a tendency to neglect this area with resources being directed only at alleviating physical morbidity ${ }^{4}$.

There were several limitations in this study. There was a lack of qualitative design such as a focus group discussion in designing the questionnaire. We adopted a cross-sectional survey design with self-administered questionnaires. Thus we were unable to comment on any causal relationship between variables studied. Reliance on self-report data has its limitations such as rating bias. However, as stress is an experience based on the perception of a mismatch between demands and resources to meet those demands, subjective self-reporting has its value in such studies. Since we only surveyed one $\mathrm{TH}$ and two $\mathrm{CHs}$, our findings may not be generalized to all hospital HCWs in Singapore or to other countries. Nevertheless, it can be expected that HCWs in other parts of the world would share these concerns and stresses.

In conclusion, this study shows that despite the majority of HCWs feeling prepared for an AI outbreak, the majority also have significant concerns such as great personal risk of exposure to the bird flu virus from their job and falling ill with the disease. TH HCWs compared to $\mathrm{CH}$ HCWs were more likely to have these concerns and perceive adverse impact of an AI pandemic on their personal life and work, possibly because of the types of patients they are caring for, as compared to $\mathrm{CH} \mathrm{HCWs}$. Healthcare institutions need to put these psychological concerns on the agenda and provide effective interventions and greater support for HCWs and their families in such future crises. TH HCWs were also found to be likelier to have participated in readiness preparation activities and this was associated with a greater proportion reporting that they and their hospital were prepared for an AI outbreak. Healthcare institutions should thus organize and provide such activities and ensure that their $\mathrm{HCW}$ s participate in such activities where indicated. In the allocation of resources for an AI pandemic, the different levels of preparedness of the type of hospital should be taken into consideration.

\section{Acknowledgements}

We would like to thank the healthcare workers for participating in our survey, and for being at the forefront in the daily battle against infectious diseases.

\section{References}

1) Koh D, Lim MK, Chia SE, Ko SM, Qian F, Ng V, Tan BH, Wong KS, Chew WM, Tang HK, Ng W, Muttakin Z, Emmanuel S, Fong NP, Koh G, Kwa CT, Tan KB, Fones C (2005) Risk perception and impact of Severe Acute Respiratory Syndrome (SARS) on work and personal lives of healthcare workers in Singapore: what can we learn? Med Care 43, 676-82.

2) Paton NI, Leo YS, Zaki SR, Auchus AP, Lee KE, Ling AE, Chew SK, Ang B, Rollin PE, Umapathi T, Sng I, Lee CC, Lim E, Ksiazek TG (1999) Outbreak of Nipah-virus infection among abattoir workers in Singapore. Lancet 354, 1253-6.

3) Goh KT, Cutter J, Heng BH, Ma S, Koh BK, Kwok C, Toh CM, Chew SK (2006) Epidemiology and control of SARS in Singapore. Ann Acad Med Singapore 35, 301-16.

4) Tam CW, Pang EP, Lam LC, Chiu HF (2004) Severe acute respiratory syndrome (SARS) in Hong Kong in 2003: stress and psychological impact among frontline healthcare workers. Psychol Med 34, 1197-204.

5) World Health Organization (WHO) (2003) Summary of probable SARS cases with onset of illness from 1 November 2002 to 31 July 2003. http://www.who.int/csr/sars/country/ table2004_04_21/en/. Accessed January 13, 2007.

6) Koh D, Lim MK, Chia SE (2003) SARS: health care work can be hazardous to health. Occup Med (Lond) 53, 241-3.

7) Verma S, Mythily S, Chan YH, Deslypere JP, Teo EK, Chong SA (2004) Post-SARS psychological morbidity and stigma among general practitioners and traditional Chinese medicine practitioners in Singapore. Ann Acad Med Singapore 33, 743 8.

8) Lim MK (2006) Integrating acute and step-down elderly care. Health Policy Monitor. http://www.hpm.org/survey/sg/a8/2. Accessed March 12, 2007.

9) Yap LK, Ow KH, Hui JY, Pang WS (2002) Premature discharge in a community hospital. Singapore Med J 43, 470 5.

10) Shaw KA, Chilcott A, Hansen E, Winzenberg T (2006) The GP's response to pandemic influenza: a qualitative study. Fam Pract 23, 267-72.

11) Qureshi K, Gershon RR, Sherman MF, Straub T, Gebbie E, McCollum M, Erwin MJ, Morse SS (2005) Health care workers' ability and willingness to report to duty during catastrophic disasters. J Urban Health 82, 378-88.

12) Balicer R, Omer S, Barnett D, Everly G (2006) Local public health workers' perceptions toward responding to an influenza pandemic. BMC Public Health 6, 99. http:// www.biomedcentral.com/1471-2458/6/99. Accessed October 
5, 2007.

13) Ruderman C, Tracy CS, Bensimon CM, Bernstein M, Hawryluck L, Shaul RZ, Upshur RE (2006) On pandemics and the duty to care: whose duty? who cares? BMC Med Ethics 7, 5. http://www.biomedcentral.com/1472-6939/7/5. Accessed October 5, 2007.

14) Maunder R, Hunter J, Vincent L, Bennett J, Peladeau N, Leszcz M, Sadavoy J, Verhaeghe LM, Steinberg R, Mazzulli $\mathrm{T}$ (2003) The immediate psychological and occupational impact of the 2003 SARS outbreak in a teaching hospital. CMAJ 168, 1245-51.

15) Nickell LA, Crighton EJ, Tracy CS, Al-Enazy H, Bolaji Y, Hanjrah S, Hussain A, Makhlouf S, Upshur RE (2004)
Psychosocial effects of SARS on hospital staff: survey of a large tertiary care institution. CMAJ 170, 793-8.

16) Lee VJ, Fernandez GG, Chen MI, Lye D, Leo YS (2006) Influenza and the pandemic threat. Singapore Med J 47, 463 70.

17) Ministry of Health Singapore (2005) Influenza Pandemic Readiness and Response Plan. www.moh.gov.sg/cmaweb/ attachments/topic/36c564c023S0/MainDec05.pdf. Accessed March 11, 2007.

18) Hogg W, Huston P, Martin C, Soto E (2006) Enhancing public health response to respiratory epidemics. Are family physicians ready and willing to help? Can Fam Physician 52, 1254-60. 